\title{
Association of Expression Levels of Clock Genes and Autophagy-Related Genes under Abnormal Light/Dark Cycle Stimulation in NAFLD Mice
}

\section{Zhu Zhu}

Zhengzhou University First Affiliated Hospital

\section{Zhengyang Wang}

Zhengzhou University First Affiliated Hospital

\section{Bo Qin}

Zhengzhou University First Affiliated Hospital

\section{Songfeng Zhao}

Zhengzhou University First Affiliated Hospital

\section{Huafei Wang}

Zhengzhou University First Affiliated Hospital

\section{Lulu He}

Zhengzhou University First Affiliated Hospital Jin Xie

Zhengzhou University First Affiliated Hospital

\section{Long Qiu}

Zhengzhou University First Affiliated Hospital

\section{Yanran Wang}

Zhengzhou University First Affiliated Hospital

\section{Li Wang}

Zhengzhou University First Affiliated Hospital

\section{Bing Chen}

Zhengzhou University First Affiliated Hospital

\section{Peipei Liu}

Zhengzhou University First Affiliated Hospital

Jinlin Liu

Jilin University

\section{Xin Tian ( $\nabla$ tianx@zzu.edu.cn )}

Zhengzhou University First Affiliated Hospital https://orcid.org/0000-0002-6080-2924

\section{Research}


Keywords: Clock Genes, Autophagy-Related Genes, Non-alcoholic fatty liver disease (NAFLD), Rev-erba Posted Date: November 24th, 2020

DOI: https://doi.org/10.21203/rs.3.rs-112997/v1

License: (c) (1) This work is licensed under a Creative Commons Attribution 4.0 International License. Read Full License 


\section{Abstract}

Background: Environmental disorders of the circadian rhythms can lead to metabolism-related diseases or exacerbate pathological conditions. Non-alcoholic fatty liver disease (NAFLD) has emerged with a growing occurrence. In the present study, we attempted to indicate whether circadian clock may influence lipid deposition and the expression levels of autophagy-related genes in liver of mice.

Methods: High-fat diet and abnormal light/dark cycles were employed to induce a mouse model of NAFLD with circadian rhythm sleep disorder. Herein, liver samples were obtained at ZT0, ZT4, ZT8, ZT12, ZT16, and ZT20 time-point to detect the rhythmic expressions of circadian genes, autophagy-related genes, and Rev-erba.

Results: Abnormal exposure to light aggravated lipid deposition in liver of mice and exacerbated disorders related to 24-h expression levels of clock genes, autophagy-related genes, and Rev-erba. Besides, Rev-erba could transcriptionally control the expression levels of autophagy-related genes.

Conclusions: The long-term high-fat diet combined with abnormal light/dark cycle stimulation aggravated the development of NAFLD and disturbed the expressions levels of autophagy-related genes. An abnormal circadian expression may lead to NAFLD aggression. Besides, the abnormal expression levels of clock genes may create an association between circadian rhythm sleep disorder and autophagy.

\section{Background}

Circadian clocks, which are intrinsic and time-tracking systems in different living organisms, can adjust behavior and physiology to adapt environmental changes during $24 \mathrm{~h}$ [1]. Circadian clocks exist in almost all of the organs and tissues, and are divide into master clock and peripheral clocks. The hypothalamic suprachiasmatic nucleus (SCN) clock is master clock, which can function autonomously. The SCN clock can reset by external light cues. A 24-h light/dark cycle may be a principal regulator that is unambiguously synchronized to Zeitgeber time (A German word termed external cues of the endogenous clock)[2]. Peripheral clocks, including liver clock, heart clock, fat clock, etc., are synchronized by the SCN clock to make temporally coordinated physiology [3]. The fundamental mechanisms of circadian rhythms are similar in the master clock and peripheral clocks, which are regulated by a series of interplaying clock genes at the transcriptional and post-translational feedback loops. Circadian locomotor output cycles kaput (Clock) and brain and muscle encoding Arnt-like protein 1 (Bmal1) form heterodimers, promoting the expression levels of downstream genes. Conversely, the period (Per1, Per2, and Per3) and cryptochrome (Cry1 and Cry2) inhibit the clock output [3]. The circadian clock genes can produce metabolic rhythms and regulate metabolic processes (e.g., lipid metabolism).

Non-alcoholic fatty liver disease (NAFLD) is the most frequent cause of liver diseases and a more potential for end-stage liver disease both in developed and in developing countries [4]. It is associated with excessive triglyceride (TG) level, which is taken as a systemic lipid metabolic disorder into account $[5,6]$. Autophagy is a conservative process in eukaryotes, and using this process, cytoplasmic lipid 
droplets are isolated in double membrane vesicles and transport to lysosomes for degradation [7]. It regulates intracellular lipid stores. Singh et al. demonstrated that autophagy could be repressed in cultured hepatocytes, promoting TG storage in lipid droplets [8]. Numerous scholars pointed out that defective hepatic autophagy is closely associated with NAFLD [8-10].

To date, NAFLD has affected within $30 \%$ of the global population, and it has emerged with a growing occurrence $[11,12]$. In order to explore the origin of rapid increase in the incidence of NAFLD, we concentrated on circadian clocks in the current study. Since the human life style has dramatically changed worldwide during the past decades, and modern life style is globally forcing more and more people to enter the asynchrony state, it is highly essential to find out an association between the social time and the internal circadian clock. Notably, environmental disorders of the circadian rhythms can lead to metabolism-related diseases or exacerbate pathological conditions. Genome-wide gene expression studies demonstrated that the circadian clock plays a significant role in liver physiology $[13,14]$. The liver clock regulates hepatic metabolism, including fatty acid, lipid, glucose, etc. [15, 16]. In addition, autophagy activity and autophagy-related genes showed daily rhythms in mice [17]. The ULK1, a critical positive regulator for autophagy initiation, is an autophagy-related gene. It maintains its rhythmicity both in zebrafish liver and mice liver [17, 18], and it controls the lipid metabolism in adipocytes [19]. Besides, microtubule-associated protein light chain 3 (LC3) is a homologue of yeast Atg8p and is associated with the autophagosome membranes. Cytosolic LC3-I and membrane-bound LC3-II are two forms of LC3. LC3II is associated with the number of autophagosomes, playing a significant circadian oscillatory role in liver and other tissues of mice, including heart, kidney, and skeletal muscle [17, 20]. It is involved in autophagosome formation, and it is transcriptionally regulated Bmal1. In addition, Atg5 is an essential molecule for autophagosome formation, and its rhythmic expression levels can be found in wild-type flies [21]. Meanwhile, inhibition of the Atg5 in hepatocytes could increase TG level [8]. Lamp1, a lysosomalassociated membrane protein 1, is involves in intracellular lipid autophagic clearance [8]. Moreover, the rhythmic expression of lamp1 was found in the liver of mice and in human retinal pigment epithelial cells $[22,23]$.

However, whether circadian rhythm disruption leads to disruption of autophagy and ultimately affects the occurrence and development of NAFLD. In the present study, we utilized abnormal light/dark cycle to induce mice circadian asynchrony. Additionally, we assessed the degree of lipid deposition in the liver of mice and detected 24-h expression levels of circadian clock genes and autophagy-related genes in the liver of mice. Besides, we preliminary evaluated how abnormal expressions of circadian clock genes could lead to changes in expressions of autophagy-related genes, and this may ultimately lead to the occurrence and development of NAFLD.

\section{Materials And Methods}

\section{A mouse model of NAFLD}


We adopted 72 8-week-old C57 BL/6J mice, which were provided by Laboratory Animal Center of Chinese Academy of Sciences (Shanghai, China). Since the high fat diet is more likely cause TG accumulation in liver [24], we used the Western-type diet (containing $0.15 \%$ cholesterol and $21 \%$ fat) for feeding mice for 6 weeks under light/dark cycle leading to circadian rhythm sleep disorder in mice [25]. All mice were randomly divided into three groups: (1) mice that were subjected to normal diet and regular light/dark cycle (C57 ND group); (2) mice that received high-fat diet under normal light/dark cycle (C57 WD group); (3) mice that received high-fat diet under abnormal light/dark cycle (C57 WD + DD/LD group).

\section{Cultivation Of Primary Hepatocytes}

Primary hepatocytes were isolated from C57 ND mice in our vitro experiments. The cells were collected according to the collagenase perfusion method [26]. Arterial blood of the mouse liver was blocked and replaced by $5 \mathrm{ml} / \mathrm{min}$ EDTA solution (including $5 \mathrm{mM}$ glucose, $0.5 \mathrm{mM}$ EDTA, $4.15 \mathrm{mM} \mathrm{NaHCO}, 136 \mathrm{mM}$ $\mathrm{NaCl}, 5.4 \mathrm{mM} \mathrm{KCl}, 0.65 \mathrm{mM} \mathrm{NaH} 2 \mathrm{PO} 4,0.85 \mathrm{mM} \mathrm{Na} 2 \mathrm{HPO}$, and $1 \mathrm{mM}$ HEPES) for $6 \mathrm{~min}$. Then, the liver was perfused with $1 \mathrm{mg} / \mathrm{ml}$ collagenase type IV. The liver was isolated and cut into pieces. Tissue fragments were filtered, centrifuged, and washed. The cells were collected and incubated in William's $E$ medium, containing $10 \%$ fetal bovine serum (FBS). After $4 \mathrm{~h}$, the old medium was replaced with a new William's E medium, containing $10 \%$ FBS to remove the dead cells. These cells at passage 0 were directly utilized herein.

\section{Short Interfering RNA (SIRNA) Transfection}

The Rev-erba siRNA was synthesized at RiboBio Co., Ltd. (Guangzhou, China). Besides, $100 \mathrm{nM}$ siRNA (Rev-erba siRNA or Control siRNA) and 50 nM LipofectamineTM 3000 (Thermo Fisher Scientific, Waltham, MA, USA) were incubated at room temperature for $5 \mathrm{~min}$. The Rev-erba siRNA or Control siRNA were mixed with LipofectamineTM 3000 for 15 min to promote the formation of Rev-erba siRNA/Lipofectamine complex and Control siRNA/Lipofectamine complex. Then, these complexes were added into the serum-free William's E medium, incubated for $6 \mathrm{~h}$. Replace with a fresh William's $\mathrm{E}$ medium for another $48 \mathrm{~h}$. The interference was detected via quantitative reverse transcription polymerase chain reaction (RT-qPCR).

\section{Histological Analysis}

Histological examination was performed to assess fatty liver degeneration in mice. The liver samples were fixed in 1\% paraformaldehyde (Sigma-Aldrich, St. Louis, MO, USA) for $24 \mathrm{~h}$. Then, the samples were embedded into paraffin and processed routinely. Finally, the samples were stained with hematoxylin and eosin (H\&E) as described previously [27].

\section{RNA Extraction And Reverse Transcription}

Total RNA was extracted from liver tissues using TRIzol reagent (Invitrogen, Carlsbad, CA, USA) according to manufacturer's instructions. RNA concentration was determined by UV spectrophotometry. RNA 
integrity was assessed using agarose gel electrophoresis. Then, $1 \mu \mathrm{g}$ RNA was reversely transcribed into cDNA using ReverTra Ace qPCR RT kit (Toyobo, Osaka, Japan) according to manufacturer's protocol.

\section{RT-QPCR}

The cDNA was amplified by ABI QuantStudio5 (Applied Biosystems, Foster City, CA, USA). The qPCR reaction mixture included $2.5 \mu \mathrm{l} \mathrm{cDNA}, 1 \mu \mathrm{l}$ of $100 \mathrm{nM}$ upstream primers, $1 \mu \mathrm{l}$ of $100 \mathrm{nM}$ downstream primers, $10 \mu$ S SYBR Green Master mix (Bio-Rad Laboratories, Hercules, CA, USA), and $20 \mu \mathrm{H} 2 \mathrm{O}$. The relative expression levels of target genes were calculated using the $2 \Delta \Delta \mathrm{Ct}$ method and normalized to 18S. The qPCR primers are presented in Table 1. 
Table 1

The primer sequences.

\begin{tabular}{|c|c|c|}
\hline Gene names & Primers & Primer sequences $\left(5^{\prime}-3^{\prime}\right)$ \\
\hline \multicolumn{3}{|c|}{ Real time-PCR } \\
\hline \multirow[t]{2}{*}{ Bmal1 } & Sense & CACTGACTACCAAGAAAGTATG \\
\hline & Antisense & АTCCATCTGCTGCCCTGAGA \\
\hline \multirow[t]{2}{*}{ Clock } & Sense & ATGAGCACCAAGACCATTCC \\
\hline & Antisense & GCTTCAGTGCTCCCAACTTC \\
\hline \multirow[t]{2}{*}{ Per2 } & Sense & CAGACTCATGATGACAGAGG \\
\hline & Antisense & GAGATGTACAGGATCTTCCC \\
\hline \multirow[t]{2}{*}{ Cry2 } & Sense & GCTAGAGTGACGGAGATGCC \\
\hline & Antisense & АCTACCACCTCACTGGGACA \\
\hline \multirow[t]{2}{*}{ ULK1 } & Sense & TGGGGAGAAGGTGTGTA \\
\hline & Antisense & TACTCTACAACAAGGGGCACA \\
\hline \multirow[t]{2}{*}{ LC3区 } & Sense & CCCAGTGATTATAGAGCGATACAAGGGGGAG \\
\hline & Antisense & CTGCAAGCGCCGTCTGATTATCTTGATGAG \\
\hline \multirow[t]{2}{*}{ Atg5 } & Sense & GGCACACCCCTGAAATGGCATTATCC \\
\hline & Antisense & ССTCAACCGCATCCTTGGATGGAC \\
\hline \multirow[t]{2}{*}{ Lamp1 } & Sense & GCCCTGGAATTGCAGTTTGG \\
\hline & Antisense & TGCTGAATGTGGGCACTAGG \\
\hline \multirow[t]{2}{*}{ Rev-erb a } & Sense & TACATTGGCTCTAGTGGCTCC \\
\hline & Antisense & CAGTAGGTGATGGTGGGAAGTA \\
\hline \multirow[t]{2}{*}{ GAPDH } & Sense & ACAGCCGCATCTTCTTGTGCAGTA \\
\hline & Antisense & GGCCTTGACTGTGCCGTGAATTT \\
\hline \multicolumn{3}{|l|}{ ChIP } \\
\hline \multirow[t]{2}{*}{ Arbp } & Sense & GAGGTGGCTTTGAACCAGAG \\
\hline & Antisense & TCTTTGTCTCTGTCTCGGAAAA \\
\hline \multirow[t]{2}{*}{ Bmal1 } & Sense & GGAAAGTAGGTTAGTGGTGCGAC \\
\hline & Antisense & AAGTCCGGCGCGGGTAAACAGG \\
\hline ULK1 & Sense & GCCTGGACTACAGGAAACCC \\
\hline
\end{tabular}




\begin{tabular}{|lll|}
\hline Gene names & Primers & Primer sequences $\left(\mathbf{5}^{\prime} \mathbf{3}^{\prime}\right.$ ) \\
\hline \multirow{2}{*}{ Atg5 } & Antisense & AACTGGCTGGCTTCAGACTC \\
& Sense & GCTGCTGACAGAGCAAAGTG \\
RNA interfering & Antisense & GGGTTTGAGACAAGCTCTCG \\
mRev-erba siRNA & Sense & CUUCGUUGUUCAACGUGAATT \\
& Antisense & UUCACGUUGAACAACGAAGTT \\
\hline
\end{tabular}

\section{Chromatin Immunoprecipitation (CHIP) Assay}

The ChIP assay was conducted using an EZ-ChIP ${ }^{\text {TM }}$ kit (Millipore, Billerica, MA, USA) according to manufacturer's instructions. The liver tissue was collected at ZT8, cut into $1 \sim 3 \mathrm{~mm} 3$ pieces, and moved into a $50 \mathrm{ml}$ centrifuge tube. Cross-link proteins to DNA by adding $27 \mu \mathrm{L}$ of $37 \%$ formaldehyde to the sample and incubate for $15 \mathrm{~min}$ at room temperature with shaking. Add glycine to a final concentration of $125 \mathrm{mM}$ to the medium and incubate with shaking for $5 \mathrm{~min}$ at room temperature. Rinse cells twice with $10 \mathrm{~mL}$ cold phosphate-buffered saline (PBS). Add $5 \mathrm{~mL}$ of cold PBS, scrape dishes thoroughly with a cell scraper and transfer into $50 \mathrm{~mL}$ tube. Add $3 \mathrm{~mL}$ PBS to dishes, scrape again and transfer the remainder of the cells to the $50 \mathrm{~mL}$ tube. Centrifuge at $4{ }^{\circ} \mathrm{C}$ for $5 \mathrm{~min}$ at $1,000 \mathrm{rpm}$. Carefully aspirate off supernatant and resuspend the pellet in ChIP lysis buffer, and incubate for $10 \mathrm{~min}$ on ice. The chromatin solutions were precipitated with $5 \mu \mathrm{g}$ Rev-erba antibody (Cell Signaling Technology Inc., Danvers, MA, USA). Each reaction was performed at $4{ }^{\circ} \mathrm{C}$ for $12 \mathrm{~h}$. Adopt Protein A Agarose to capture Rev-erba complex. Elute the complex from Rev-erba antibody and reverse protein-DNA crosslinks. The qPCR was performed to assess the enrichment of Rev-erba protein.

\section{Statistical Analysis}

The data were presented as mean \pm standard deviation (SD). Single cosinor method was employed to analyze circadian rhythm as previously described $[28,29]$. The following equation was formulated for cosine function: $Y(t)=M+A^{*} \cos \left(x^{*} t+\mu\right)$. The daily rhythm characteristics included mesor (midline estimating statistic of rhythm corresponding to the mean level), amplitude (half of the peak-to-trough difference of the fitted cosine function), and acrophase (the crest time of rhythm given in degrees $\left({ }^{\circ} \mathrm{C}\right)$, where $360^{\circ} \mathrm{C}$ is corresponding to a $24-\mathrm{h}$ cycle) were estimated by the above-mentioned function. Differences between the values of each pair of parameters were compared by one-way analysis of variance (ANOVA), and $P \leq 0.05$ was considered statistically significant.

\section{Results}




\section{The abnormal lipid accumulation in hepatic cells after undergoing high-fat diet and abnormal light/dark cycle stimulation}

To assess whether high-fat diet-induced NAFLD in mice, hematoxylin and eosin (H\&E) staining was performed to evaluate lipid accumulation in the liver cells. As displayed in Fig. 1, there was no abnormal lipid accumulation in control mice that received normal diet under regular light/dark cycle. However, for mice that received high-fat diet for 6 weeks, lipid accumulation was noted in several liver cells. According to entrainment of the circadian system under light/dark cycle [30], we herein conducted the experiments on the base of high-fat diet and abnormal light/dark cycle stimulation. Figure 1 illustrates under high-fat diet and abnormal light/dark cycle, more lipid droplets were accumulated in the liver cells. The abovementioned results suggested that high-fat diet and abnormal light/dark cycle could aggravate the fatty liver degeneration in mice.

\section{The rhythmic expression levels of circadian genes in liver tissues}

Circadian clock genes can modulate liver lipid metabolism. Therefore, the rhythmic expression levels of clock genes (Bmal1, Clock, Per2, and Cry2) were detected in C57 ND, C57 WD, and C57 WD + LD/DD groups at ZT0, ZT4, ZT8, ZT12, ZT16 and ZT20 (Table 2). As shown in Fig. 2, the circadian oscillation of Bmal1 in C57 WD + LD/DD group was attenuated. The expression level of Per2 was significantly increased in C57 WD + LD/DD group compared with that in C57 ND group. The mesors of Per2 were remarkably elevated in C57 WD + LD/DD group compared with those in control group. Moreover, the amplitudes of Per2 were notably increased in C57 WD + LD/DD group compared with those in C57 ND group. Furthermore, the peak periodic values of Clock, Per2, and Cry2 were altered in C57 WD + LD/DD group compared with those in C57 ND group. 
Table 2

Circadian rhythmic parameters of clock genes,ULK1, LC3 II, Atg5 and Lamp1 in mouse liver.

\begin{tabular}{|llll|}
\hline Gene & Mesor & Amplitude & Acrophase ZT (h) \\
\hline C57 ND & & & \\
\hline Bmal1 & $1.07 \pm 0.24$ & $0.57 \pm 0.03$ & $22.17 \pm 0.34$ \\
\hline Clock & $1.09 \pm 0.30$ & $0.61 \pm 0.17$ & $21.40 \pm 0.13$ \\
\hline Per2 & $2.93 \pm 0.82$ & $2.85 \pm 0.54$ & $10.88 \pm 0.12$ \\
\hline Cry2 & $2.83 \pm 0.91$ & $2.01 \pm 0.40$ & $8.54 \pm 0.08$ \\
\hline ULK1 & $0.85 \pm 0.31$ & $0.76 \pm 0.27$ & $1.88 \pm 0.07$ \\
\hline LC3 II & $1.25 \pm 0.08$ & $0.97 \pm 0.36$ & $21.05 \pm 0.17$ \\
\hline Atg5 & $2.19 \pm 1.05$ & $2.17 \pm 0.28$ & $17.89 \pm 1.77$ \\
\hline Lamp1 & $5.79 \pm 1.24$ & $6.85 \pm 3.98$ & $9.54 \pm 0.04$ \\
\hline Rev-erb a & $1.20 \pm 0.33$ & $0.94 \pm 0.33$ & $4.15 \pm 0.04$ \\
\hline C57 WD & & & \\
\hline Bmal1 & $0.99 \pm 0.24$ & $0.67 \pm 0.20$ & $19.32 \pm 0.14$ \\
\hline Clock & $0.67 \pm 0.18$ & $0.50 \pm 0.09$ & $21.28 \pm 0.06$ \\
\hline Per2 & $4.43 \pm 1.89$ & $3.33 \pm 1.15$ & $11.27 \pm 0.12$ \\
\hline Cry2 & $2.68 \pm 1.12$ & $1.90 \pm 0.76$ & $8.92 \pm 0.12$ \\
\hline ULK1 & $0.77 \pm 0.43$ & $0.63 \pm 0.32$ & $1.96 \pm 0.22$ \\
\hline LC3 II & $0.95 \pm 0.11 * *$ & $0.91 \pm 0.66$ & $21.24 \pm 0.05$ \\
\hline Atg5 & $1.56 \pm 0.58$ & $1.41 \pm 0.21 *$ & $17.65 \pm 0.05$ \\
\hline Lamp1 & $4.33 \pm 1.81$ & $4.16 \pm 1.60 *$ & $9.85 \pm 0.09$ \\
\hline Rev-erb a & $1.30 \pm 0.37$ & $1.07 \pm 0.34$ & $2.62 \pm 1.47$ \\
\hline C57 WD DD/DL & & & \\
\hline Bmal1 & - & & \\
\hline Clock & & & \\
\hline Per2 & & & \\
\hline Cry2 & & & \\
\hline
\end{tabular}




\begin{tabular}{|llll|}
\hline Gene & Mesor & Amplitude & Acrophase ZT (h) \\
\hline ULK1 & - & - & - \\
\hline LC3 II & - & - & - \\
\hline Atg5 & $2.63 \pm 0.91$ & $1.54 \pm 0.49$ & $12.27 \pm 0.08^{\star \star}$ \\
\hline Lamp1 & $2.58 \pm 0.27^{\star \star}$ & $2.19 \pm 0.92^{\star \star}$ & $16.04 \pm 0.25^{\star \star}$ \\
\hline Rev-erb a & $0.38 \pm 0.18^{\star \star}$ & $0.35 \pm 0.16^{\star}$ & $0.50 \pm 0.07^{\star \star}$ \\
\hline $\mathrm{n}=4 .{ }^{*}, \mathrm{p}<0.05,{ }^{* \star}, \mathrm{p}<0.01$ versus C57 ND mice & \\
\hline
\end{tabular}

\section{Circadian rhythmic expression patterns of autophagy-related genes in liver tissues}

Autophagy is a highly conserved intracellular degradation system, and recently was shown to display circadian rhythms in mice. In the present study, we detected the expression levels of autophagy-related genes (ULK1, LC3囚, Atg5, and Lamp1) in liver tissues of mice (Table 2). As shown in Fig. 3, ULK1 and LC3 $\triangle$ in C57 WD + LD/DD mice were lost its circadian rhythms. The mesors of LC3 $\triangle$ in C57WD group and Lamp1 in C57 WD + LD/DD group were decreased compared with those in C57 ND group. Additionally, the amplitudes of Atg5 in C57 WD group were significantly attenuated compared with those in C57 ND group. Besides, the amplitudes of Lamp1 in both C57 WD and C57 WD + LD/DD groups were markedly reduced compared with those in control group. Moreover, the peak values of Atg5 and Lamp1 were dramatically decreased in C57 WD and C57 WD + LD/DD groups, respectively, compared with those in control group. Meanwhile, the peak periodic values of Atg5 and Lamp1 in C57 WD + LD/DD groups were different from those in control group.

\section{Diurnal expression of Rev-erba gene and its relationship with autophagy-related genes}

The mechanism of the abnormal light/dark cycle stimulation causing changes in the circadian rhythm of autophagy-related genes have not been fully elucidated. The circadian clock gene Rev-erba, known as nuclear receptor 1D1, plays a circadian oscillatory role in liver, heart, and skeletal muscle. Notably, Reverba regulates the expression levels of several circadian target genes and plays regulatory roles in lipid metabolism [31]. Moreover, Rev-erba deficiency resulted in deactivation of the Stk11-Ampk-Sirt1Ppargc1-a signaling pathway, whereas autophagy was up-regulated, resulting in both impaired mitochondrial biogenesis and increased clearance [32]. Additionally, Huang et al. pointed out that the circadian clock can directly regulate the expression levels of autophagy-related genes through Rev-erba in zebrafish liver [18]. In the current research, we attempted to indicate whether Rev-erba could induce changes in rhythmic expression levels of autophagy-related genes in C57 WD + LD/DD group. We first examined the expression level of Rev-erba in C57ND, C57WD and C57 WD + LD/DD groups (Table 2). As shown in Fig. 4, the mesors were significantly decreased in C57 WD + LD/DD group compared with those in C57ND group. The amplitudes of Rev-erba were markedly inhibited in C57 WD + LD/DD group than 
those in C57 ND group. Moreover, the peak periodic values in C57 WD + LD/DD group were markedly altered compared with those in control group (Fig. 4A). The rhythmic expression level of Rev-erba changed in C57WD + LD/DD group.

In order to explore the relationship between Rev-erba and autophagy-related genes, the ChIP assay was performed to indicate whether Rev-erba could transcriptionally regulate the expression levels of autophagy-related genes. However, Rev-erba promoted the expression level of Bmal1 as high as 9.3fold. Besides, Rev-erba upregulated the expression levels of autophagy-related genes (ULK1 and Atg5) within 3.45- and 4.78-fold, respectively (Fig. 4B).

To further verify the effects of Rev-erba on the expression levels of ULK1 and Atg5, siRNA was used to detect the expression levels of ULK1 and Atg5. In primary liver cells, when the expression level of Rev-erba decreased to $~ 1.56$-fold, the expression levels of ULK1 and Atg5 were reduced to $\sim 0.96$ - and $~ 1.19$-fold, respectively (Fig. 4C). The above-mentioned results indicated that the alteration of expression levels of autophagy-related genes induced by circadian rhythm sleep disorders may be accomplished through Reverba.

\section{Discussion}

In the present study, we showed that long-term high-fat diet combined with abnormal light/dark cycle stimulation may lead to or aggaravate NAFLD and change circadian expression patterns of circadian clock genes and autophagy-related genes. The pathogenic mechanisms of NAFLD have not been well understood. However, circadian clock participates in lipid and glucose metabolism, and is associated with metabolic syndrome. The relationship between the pathogenesis of NAFLD and impairment of circadian has been previously reported by a number of scholars. For instance, shift workers with irregular sleep time are more susceptible to obesity and associated disorders such as NAFLD [33]. The circadian clock genes, such as Clock, Per2, and Rev-erba regulate liver lipid metabolism, and circadian rhythm disorder leads to lipid accumulation in liver cells [34-36]. Moreover, a regular eating time and a proper sleep time can reduce the risk of metabolic syndrome and NAFLD [37]. In the present study, high-fat diet and abnormal light/dark cycle stimulation led to the accumulation of lipid and the abnormal expression levels of circadian clock genes in the liver of mice. However, the specific relationship between circadian rhythm disorder and the pathogenesis of NAFLD has not yet been fully elucidated.

Circadian rhythms are closely related to metabolism, while how such daily rhythms organize lipid metabolism in liver cells has not been fully understood. Apart from canonical lipolysis, autophagy, a highly conserved biological degradation process, contributes to lipid drops degradation. Consistent with our study, autophagy-related genes displayed circadian rhythms in both mice and in zebrafish [18, 38], and its periodic induction may provide a new association between daily rhythms and lipid metabolism. Autophagy has shown robust circadian clocks in the liver of mice, and it is accompanied by cyclic induction of autophagy-related genes involved in various steps of autophagy [17]. In the zebrafish liver, circadian rhythms directly regulate autophagy-related genes via Rev-erba [18]. The master regulators of 
autophagy, TFEB and TFE3, exhibited a circadian expression manner [39]. In the present research, Reverba showed alteration of biological rhythm during $24 \mathrm{~h}$ that resulted from nutrient and light stimulation. Furthermore, we demonstrated that Rev-erba could transcriptionally regulate the expression levels of ULK1 and Atg5. Meanwhile the changes in mRNA levels of Rev-erba, ULK1, and Atg5 were consistent with results of ChIP assay. Therefore, it can be concluded that Rev-erba may create an association between biological rhythm and autophagy, however, further research needs to be conducted to confirm this finding.

Selective autophagy termed 'lipophagy' may create a novel association between circadian clock and development and progression of NAFLD. In recent years, the function of autophagy in NAFLD has attracted scholars' attention. Liver-specific knockout of the autophagy gene Atg7 in mice has displayed a remarkable lipid accumulation, which could mimick the human NAFLD condition [8]. Treatment with activators of autophagy could lower TG level in the liver and blood [40]. Tanaka et al. demonstrated that rubicon, an autophagy-inhibiting protein, could repress autophagy and prompt lipid accumulation in hepatocyte in NAFLD [41]. The present research revealed that the greater the changes in rhythmic expression levels of autophagy-related genes, the more lipid accumulated in the liver cells.

\section{Conclusions}

In summary, the current study revealed that high-fat diet and light stimulation may result in lipid accumulation in liver cells of mice, and alter expression levels of circadian clock genes and autophagyrelated genes. Furthermore, Rev-erba may create an association between circadian rhythms and autophagy.

\section{Abbreviations}




\begin{tabular}{|c|c|}
\hline NAFLD & Non-alcoholic fatty liver disease \\
\hline $\mathrm{ZT}$ & Zeitgeber time \\
\hline Rev-erb $\alpha$ & $\begin{array}{l}\text { nuclear receptor subfamily } 1 \text {, group D, } \\
\text { member } 1\end{array}$ \\
\hline SCN & suprachiasmatic nucleus \\
\hline Clock & Circadian locomotor output cycles kaput \\
\hline Bmal1 & brain and muscle encoding Arnt-like protein 1 \\
\hline Per1 & Period1 \\
\hline Per2 & Period2 \\
\hline Per3 & Period3 \\
\hline Cry1 & Cryptochrome1 \\
\hline Cry2 & Cryptochrome2 \\
\hline TG & triglyceride \\
\hline ULK1 & unc-51 like kinase 1 \\
\hline LC3 & microtubule-associated protein light chain 3 \\
\hline $\operatorname{Atg} 5$ & autophagy related 5 \\
\hline Lamp1 & lysosomal-associated membrane protein 1 \\
\hline ND & normal diet \\
\hline WD & high-fat diet \\
\hline $\mathrm{DD} / \mathrm{LD}$ & abnormal light/dark cycle \\
\hline FBS & fetal bovine serum \\
\hline siRNA & Short interfering RNA \\
\hline RT-qPCR & $\begin{array}{l}\text { reverse transcription polymerase chain } \\
\text { reaction }\end{array}$ \\
\hline ChIP & Chromatin immunoprecipitation \\
\hline PBS & phosphate-buffered saline \\
\hline SD & standard deviation \\
\hline $\mathrm{H} \& \mathrm{E}$ & hematoxylin and eosin \\
\hline
\end{tabular}




\section{Declarations}

\section{Compliance with ethical standards}

All procedures were in accordance with the ethical standards of the institution and approved by the Animal Care and Ethics Committee of Zhengzhou University, Zhengzhou, China.

\section{Consent for publication}

Not applicable.

\section{Availability of data and material}

Not applicable.

\section{Conflicts of interest}

The authors have no competing interests to disclose.

\section{Funding}

This study was supported by the National Natural Science Foundation of China (No.81900791 and No.31801142), Henan Medical Science and Technology Research Plan Joint Co-construction Project (No. 2018020080 and No. 2018020045), Henan Science and Technology Project in 2019 (No.192102310322).Henan Key Project of Research and Development Plan (Science and technology) (No.192102310192).

\section{Author's contributions}

Study design: ZZ, ZYW, BQ and XT. Study conduct: ZZ, ZYW, BQ, SFZ, JLL and XT. Data collection: ZZ, HFW, LLH and JX. Data analysis: ZYW, LQ, YRW and LW. Data interpretation: ZZ, BC, PPL and XT. Drafting manuscript: ZZ, BQ and XT. Approving the final version of the manuscript: All authors. ZZ, ZYW, BQ and $\mathrm{XT}$ take responsibility for the integrity of the data analysis. The sponsors had no role in study design, data collection and analysis, decision to publish, or preparation of the manuscript.

\section{Acknowledgements}

We thank Dr. Jianfa Zhang for generously providing single cosinor method to analyze circadian rhythm.

\section{References}

1. Schibler U, Sassone-Corsi P: A web of circadian pacemakers. Cell 2002, 111:919-922.

2. Wever RA: Light effects on human circadian rhythms: a review of recent Andechs experiments. $J$ Biol Rhythms 1989, 4:161-185. 
3. Green CB, Takahashi JS, Bass J: The meter of metabolism. Cell 2008, 134:728-742.

4. Younossi Z, Anstee QM, Marietti M, Hardy T, Henry L, Eslam M, George J, Bugianesi E: Global burden of NAFLD and NASH: trends, predictions, risk factors and prevention. Nat Rev Gastroenterol Hepatol 2018, 15:11-20.

5. Samuel VT, Shulman GI: Nonalcoholic Fatty Liver Disease as a Nexus of Metabolic and Hepatic Diseases. Cell Metab 2018, 27:22-41.

6. Friedman SL, Neuschwander-Tetri BA, Rinella M, Sanyal AJ: Mechanisms of NAFLD development and therapeutic strategies. Nat Med 2018, 24:908-922.

7. Mizushima N, Levine B, Cuervo AM, Klionsky DJ: Autophagy fights disease through cellular selfdigestion. Nature 2008, 451:1069-1075.

8. Singh R, Kaushik S, Wang Y, Xiang Y, Novak I, Komatsu M, Tanaka K, Cuervo AM, Czaja MJ: Autophagy regulates lipid metabolism. Nature 2009, 458:1131-1135.

9. Schulze RJ, Drizyte K, Casey CA, McNiven MA: Hepatic Lipophagy: New Insights into Autophagic Catabolism of Lipid Droplets in the Liver. Hepatol Commun 2017, 1:359-369.

10. Wu WKK, Zhang L, Chan MTV: Autophagy, NAFLD and NAFLD-Related HCC. Adv Exp Med Bio/ 2018, 1061:127-138.

11. Wong RJ, Aguilar M, Cheung R, Perumpail RB, Harrison SA, Younossi ZM, Ahmed A: Nonalcoholic steatohepatitis is the second leading etiology of liver disease among adults awaiting liver transplantation in the United States. Gastroenterology 2015, 148:547-555.

12. Bonora $E$, Targher G: Increased risk of cardiovascular disease and chronic kidney disease in NAFLD. Nat Rev Gastroenterol Hepatol 2012, 9:372-381.

13. Panda S, Antoch MP, Miller BH, Su Al, Schook AB, Straume M, Schultz PG, Kay SA, Takahashi JS, Hogenesch JB: Coordinated transcription of key pathways in the mouse by the circadian clock. Cell 2002, 109:307-320.

14. Akhtar RA, Reddy AB, Maywood ES, Clayton JD, King VM, Smith AG, Gant TW, Hastings MH, Kyriacou $\mathrm{CP}$ : Circadian cycling of the mouse liver transcriptome, as revealed by CDNA microarray, is driven by the suprachiasmatic nucleus. Curr Biol 2002, 12:540-550.

15. Lee AH, Glimcher LH: Intersection of the unfolded protein response and hepatic lipid metabolism. Cell Mol Life Sci 2009, 66:2835-2850.

16. Marcheva B, Ramsey KM, Buhr ED, Kobayashi Y, Su H, Ko CH, Ivanova G, Omura C, Mo S, Vitaterna $\mathrm{MH}$, et al: Disruption of the clock components CLOCK and BMAL1 leads to hypoinsulinaemia and diabetes. Nature 2010, 466:627-631.

17. Ma D, Panda S, Lin JD: Temporal orchestration of circadian autophagy rhythm by C/EBPbeta. EMBO $J$ 2011, 30:4642-4651.

18. Huang $G$, Zhang F, Ye Q, Wang $H$ : The circadian clock regulates autophagy directly through the nuclear hormone receptor Nr1d1/Rev-erbalpha and indirectly via Cebpb/(C/ebpbeta) in zebrafish. Autophagy 2016, 12:1292-1309. 
19. Ro SH, Jung CH, Hahn WS, Xu X, Kim YM, Yun YS, Park JM, Kim KH, Seo M, Ha TY, et al: Distinct functions of Ulk1 and Ulk2 in the regulation of lipid metabolism in adipocytes. Autophagy 2013, 9:2103-2114.

20. Kabeya Y, Mizushima N, Ueno T, Yamamoto A, Kirisako T, Noda T, Kominami E, Ohsumi Y, Yoshimori T: LC3, a mammalian homologue of yeast Apg8p, is localized in autophagosome membranes after processing. EMBO J 2000, 19:5720-5728.

21. Kijak E, Pyza E: TOR signaling pathway and autophagy are involved in the regulation of circadian rhythms in behavior and plasticity of L2 interneurons in the brain of Drosophila melanogaster. PLOS One 2017, 12:e0171848.

22. Yoshitane H, Ozaki H, Terajima H, Du NH, Suzuki Y, Fujimori T, Kosaka N, Shimba S, Sugano S, Takagi T, et al: CLOCK-controlled polyphonic regulation of circadian rhythms through canonical and noncanonical E-boxes. Mol Cell Biol 2014, 34:1776-1787.

23. Milicevic N, Mazzaro N, de Bruin I, Wils E, Ten Brink J, Asbroek AT, Mendoza J, Bergen A, FelderSchmittbuhl MP: Rev-Erbalpha and Photoreceptor Outer Segments modulate the Circadian Clock in Retinal Pigment Epithelial Cells. Sci Rep 2019, 9:11790.

24. Wang D, Wei Y, Pagliassotti MJ: Saturated fatty acids promote endoplasmic reticulum stress and liver injury in rats with hepatic steatosis. Endocrinology 2006, 147:943-951.

25. Ding JM, Buchanan GF, Tischkau SA, Chen D, Kuriashkina L, Faiman LE, Alster JM, McPherson PS, Campbell KP, Gillette MU: A neuronal ryanodine receptor mediates light-induced phase delays of the circadian clock. Nature 1998, 394:381-384.

26. Li X, Hylemon P, Pandak WM, Ren S: Enzyme activity assay for cholesterol 27-hydroxylase in mitochondria. J Lipid Res 2006, 47:1507-1512.

27. Zhu Z, Xu L, Cai T, Yuan G, Sun N, Lu C, Qian R: Clock represses preadipocytes adipogenesis via GILZ. J Cell Physio/ 2018, 233:6028-6040.

28. Zhu Z, Hua B, Shang Z, Yuan G, Xu L, Li E, Li X, Sun N, Yan Z, Qian R, Lu C: Altered Clock and Lipid Metabolism-Related Genes in Atherosclerotic Mice Kept with Abnormal Lighting Condition. Biomed Res Int 2016, 2016:5438589.

29. Nelson W, Tong YL, Lee JK, Halberg F: Methods for cosinor-rhythmometry. Chronobiologia 1979, 6:305-323.

30. Albrecht U: Timing to perfection: the biology of central and peripheral circadian clocks. Neuron 2012, 74:246-260.

31. Cho H, Zhao X, Hatori M, Yu RT, Barish GD, Lam MT, Chong LW, DiTacchio L, Atkins AR, Glass CK, et al: Regulation of circadian behaviour and metabolism by REV-ERB-alpha and REV-ERB-beta. Nature 2012, 485:123-127.

32. Woldt E, Sebti Y, Solt LA, Duhem C, Lancel S, Eeckhoute J, Hesselink MK, Paquet C, Delhaye S, Shin Y, et al: Rev-erb-alpha modulates skeletal muscle oxidative capacity by regulating mitochondrial biogenesis and autophagy. Nat Med 2013, 19:1039-1046. 
33. Johnston JD: Physiological links between circadian rhythms, metabolism and nutrition. Exp Physiol 2014, 99:1133-1137.

34. Mazzoccoli G, Vinciguerra M, Oben J, Tarquini R, De Cosmo S: Non-alcoholic fatty liver disease: the role of nuclear receptors and circadian rhythmicity. Liver Int 2014, 34:1133-1152.

35. Shi D, Zhan X, Yu X, Jia M, Zhang Y, Yao J, Hu X, Bao Z: Inhibiting CB1 receptors improves lipogenesis in an in vitro non-alcoholic fatty liver disease model. Lipids Health Dis 2014, 13:173.

36. Shi D, Chen J, Wang J, Yao J, Huang Y, Zhang G, Bao Z: Circadian Clock Genes in the Metabolism of Non-alcoholic Fatty Liver Disease. Front Physiol 2019, 10:423.

37. Shetty A, Hsu JW, Manka PP, Syn WK: Role of the Circadian Clock in the Metabolic Syndrome and Nonalcoholic Fatty Liver Disease. Dig Dis Sci 2018, 63:3187-3206.

38. Ryzhikov M, Ehlers A, Steinberg D, Xie W, Oberlander E, Brown S, Gilmore PE, Townsend RR, Lane WS, Dolinay T, et al: Diurnal Rhythms Spatially and Temporally Organize Autophagy. Cell Rep 2019, 26:1880-1892 e1886.

39. Pastore N, Ballabio A: Keeping the autophagy tempo. Autophagy 2019, 15:1854-1856.

40. Lin CW, Zhang H, Li M, Xiong X, Chen X, Chen X, Dong XC, Yin XM: Pharmacological promotion of autophagy alleviates steatosis and injury in alcoholic and non-alcoholic fatty liver conditions in mice. J Hepatol 2013, 58:993-999.

41. Tanaka S, Hikita H, Tatsumi T, Sakamori R, Nozaki Y, Sakane S, Shiode Y, Nakabori T, Saito Y, Hiramatsu N, et al: Rubicon inhibits autophagy and accelerates hepatocyte apoptosis and lipid accumulation in nonalcoholic fatty liver disease in mice. Hepatology 2016, 64:1994-2014.

\section{Figures}

C57 ND

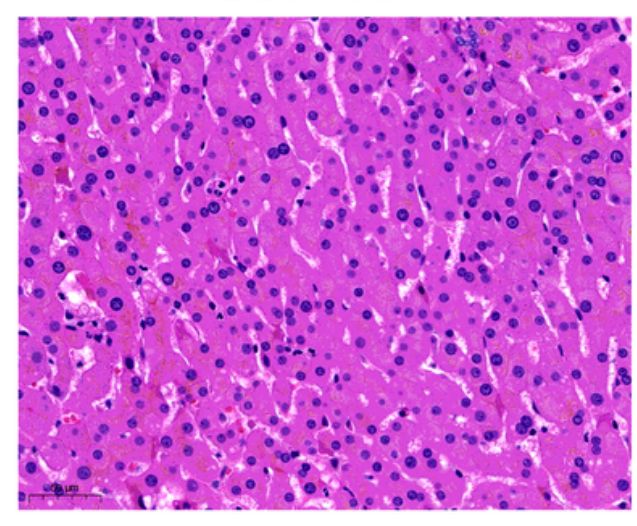

C57 WD

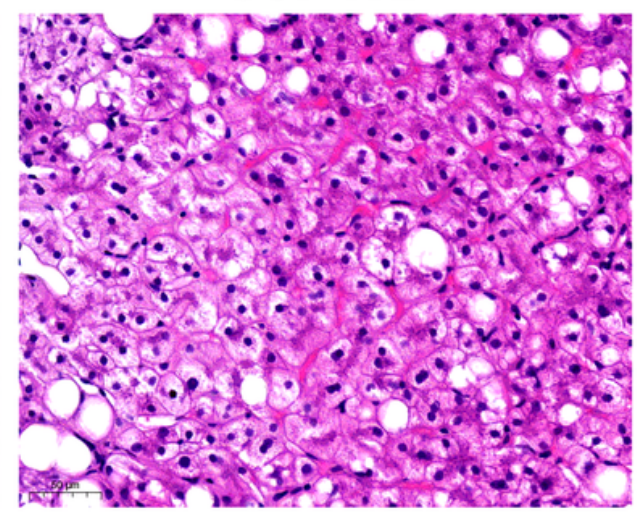

C57 WD+LD/DD

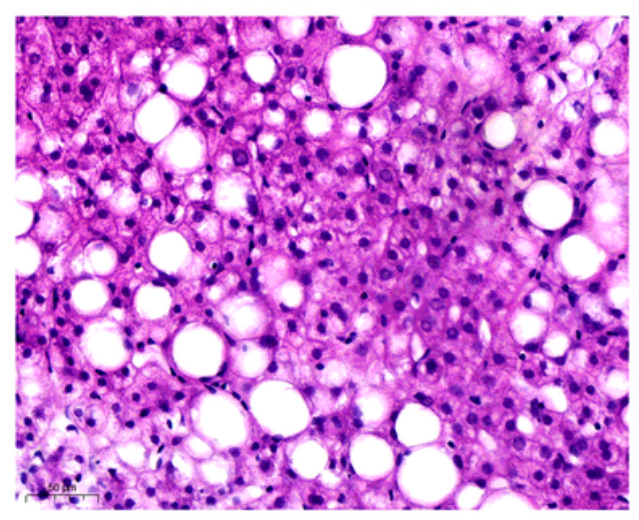

Figure 1

Representative images of hematoxylin and eosin (H\&E) staining of the liver tissues from C57 ND, C57 WD, and C57 WD+LD/DD mice. The liver tissues of C57 ND, C57 WD, and C57 WD+LD/DD mice were obtained at ZT8 time-point. We performed H\&E staining of the liver tissues, and lipid droplets were significantly 

in C57 WD+LD/DD mice compared with that in C57 WD mice (Original magnification, $\times 100$ ). All data were shown as mean \pm standard deviation (SD) ( $\mathrm{n}=4$ for each group).
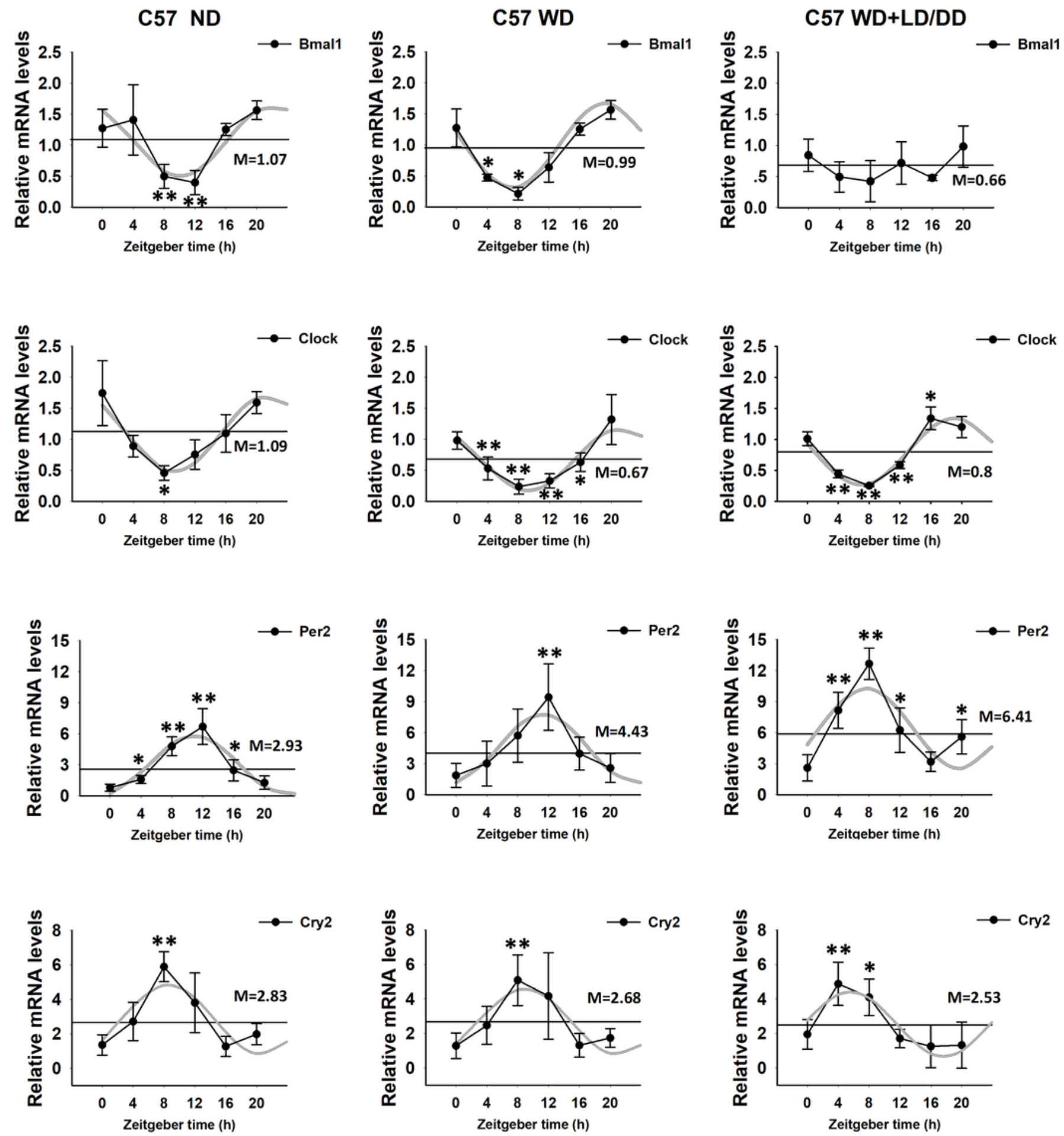

Figure 2

Expression levels of circadian clock genes in liver tissues of C57 ND, C57 WD, and C57 WD+LD/DD mice. The liver tissues of C57 ND, C57 WD, and C57 WD+LD/DD mice were obtained at ZT0, ZT4, ZT8, ZT12, 
ZT16, and ZT20 time-point. The expression levels of circadian clock genes, such as Bmal1, Clock, Per2, and Cry2 were detected by RT-qPCR. These four clock genes showed 24-h circadian oscillations in C57 ND, C57 WD, and C57 WD+LD/DD mice, except for Bmal1 in C57 WD+LD/DD mice. The expression level of Per2 was remarkably increased in C57 WD+LD/DD mice compared with that in C57 ND mice. The mesors of Per2 were increased in C57 WD+LD/DD mice compared with those in control mice. The amplitudes of Per2 were heightened in C57 WD+LD/DD mice compared with those in C57 ND mice. The peak periodic values of Clock, Per2, and Cry2 were markedly altered in C57 WD+LD/DD mice compared with those in control mice. The mRNA levels were normalized to GAPDH. All data were expressed as mean \pm SD at ZT0, ZT4, ZT8, ZT12, ZT16, and ZT20 time-point ( $\mathrm{n}=4$ for each group), $*, \mathrm{P}<0.05, * *, \mathrm{P}<$ 0.01 versus $Z T O$.

a
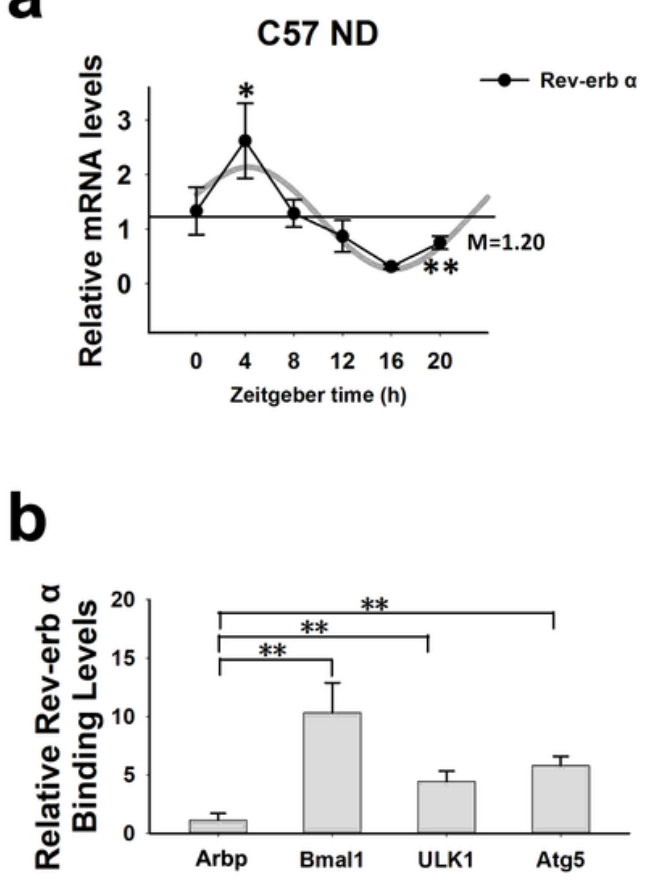
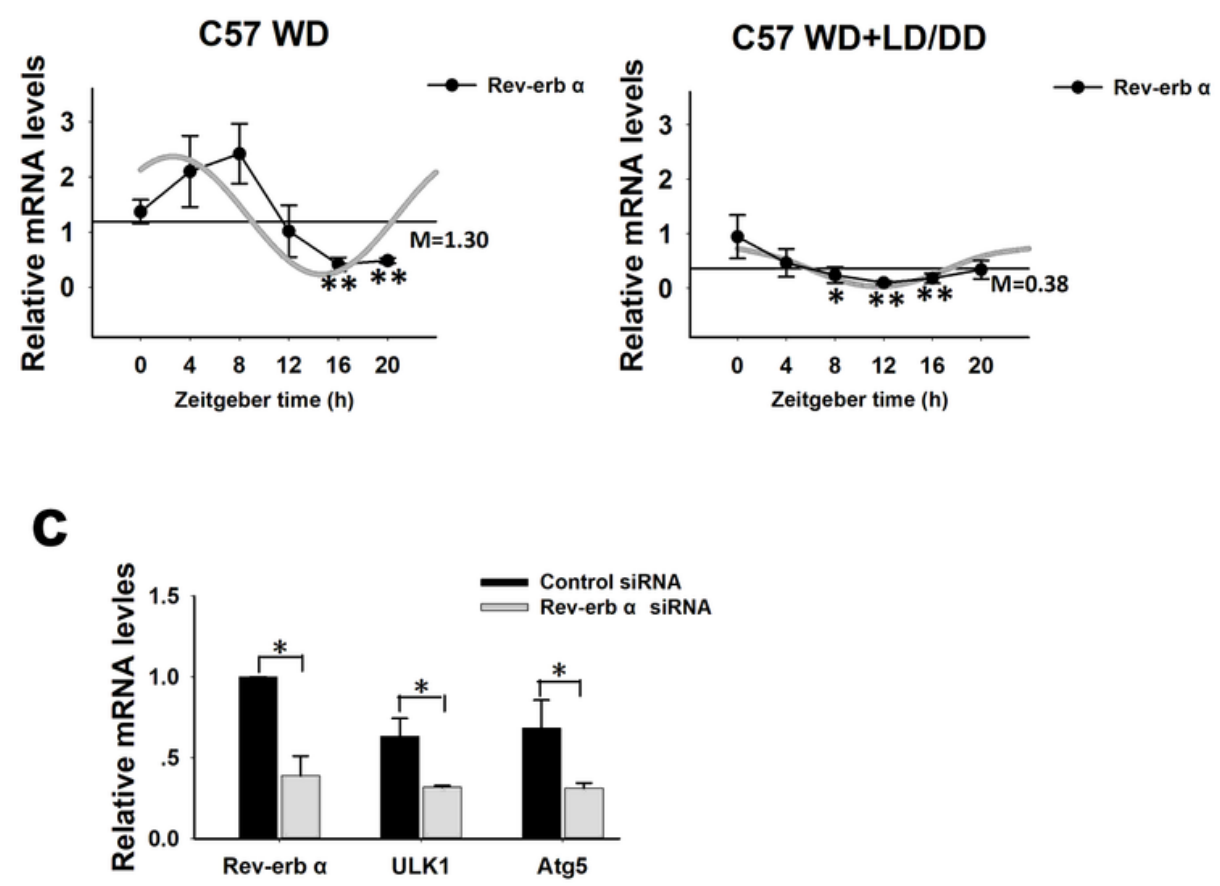

\section{Figure 3}

Circadian expression levels of autophagy-related genes in liver tissues of C57 ND, C57 WD, and C57 WD+LD/DD mice. Circadian rhythms of ULK1 and LC3囚 in C57 WD+LD/DD mice were lost. The mesors of LC3 $\$ in C57WD mice were remarkably reduced compared with those in C57 ND mice, and those of Lamp1 in C57 WD+LD/DD mice were significantly decreased compared with those in C57 ND mice. The amplitudes of Atg5 in C57 WD mice were notably repressed than those in C57 ND mice, and the amplitudes of Lamp1 in both C57 WD mice and C57 WD+LD/DD mice were remarkably reduced compared with those in control mice. The peak expression levels of Atg5 in C57 WD mice and Lamp1 in C57 WD+LD/DD mice were significantly decreased compared with those in control mice. The peak periodic values of Atg 5 in C57 WD+LD/DD mice and Lamp1 in C57 WD+LD/DD mice were markedly different from those in control mice. The mRNA levels were normalized to GAPDH. All the data were presented as mean $\pm \mathrm{SD}$ at $\mathrm{ZT0}, \mathrm{ZT} 4, \mathrm{ZT} 8, \mathrm{ZT} 12, \mathrm{ZT} 16$, and ZT20 time-point ( $\mathrm{n}=4$ for each group), $*, \mathrm{P}<$ $0.05, * *, \mathrm{P}<0.01$ versus $\mathrm{ZTO}$. 
C57 ND
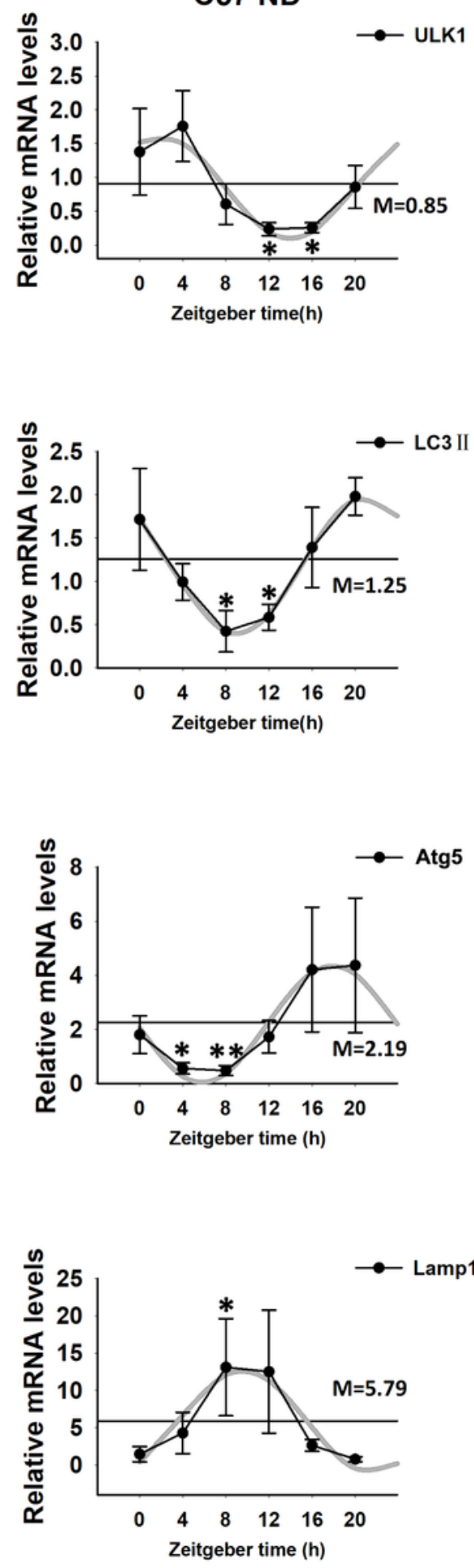

C57 WD
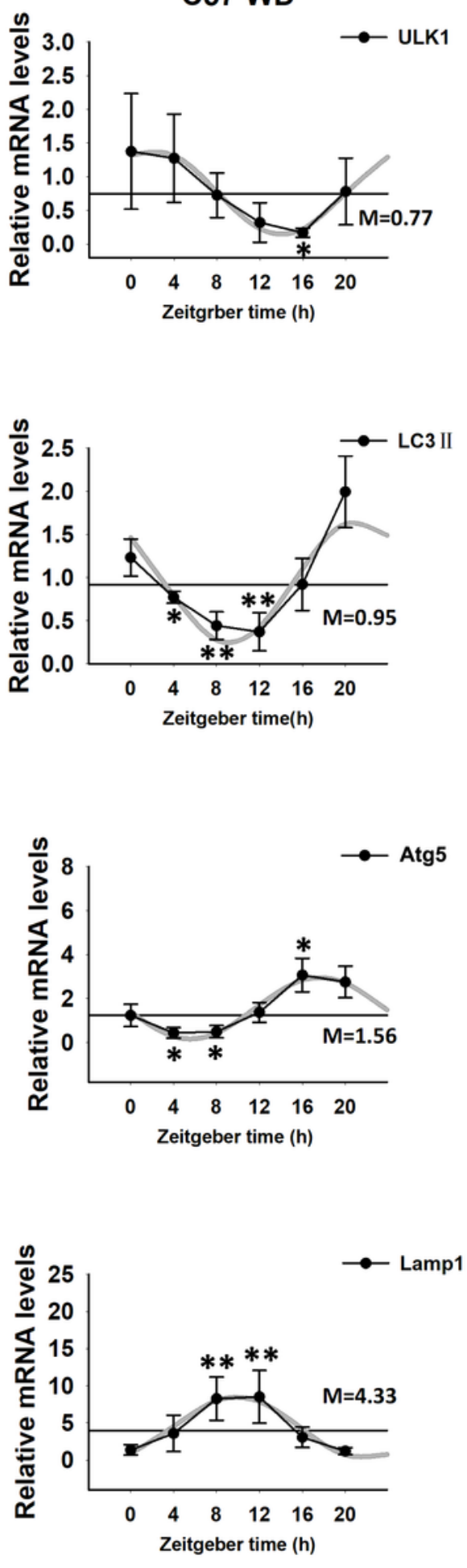

C57 WD+LD/DD
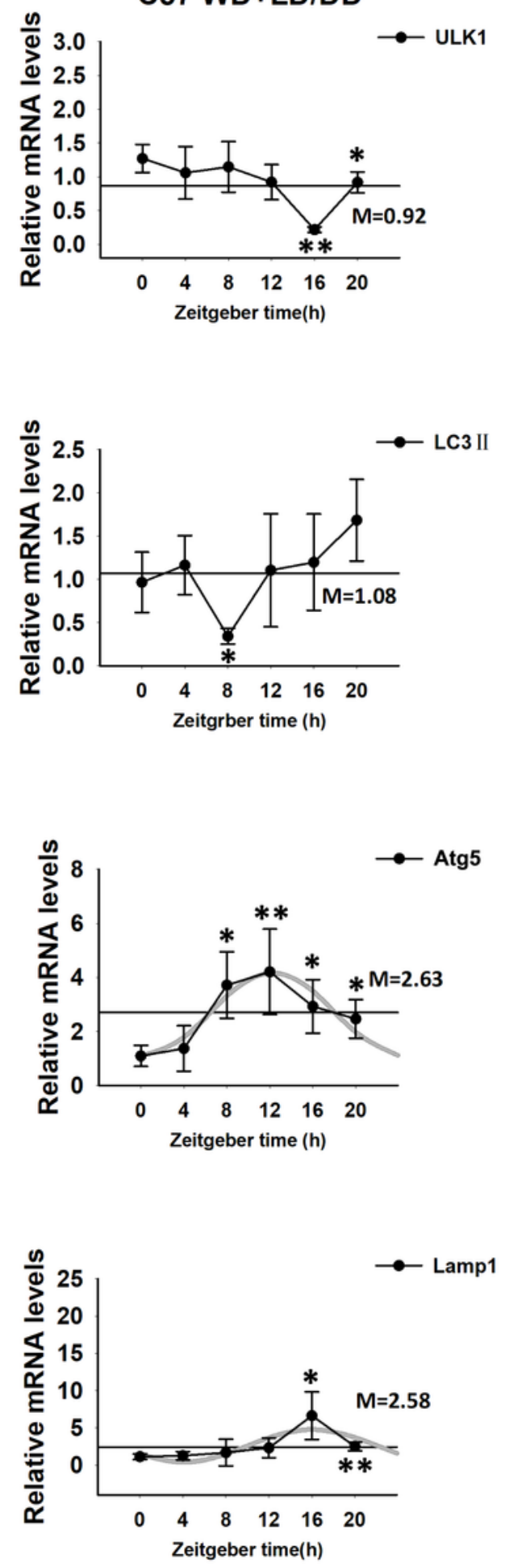

\section{Figure 4}

Circadian expression level of Rev-erba and its relationship with autophagy-related gene. (A) 24-h expression levels of Rev-erba in C57ND, C57WD, and C57 WD+LD/DD mice. The mesors in C57 $W D+L D / D D$ mice were significantly decreased compared with those in C57 ND mice. The amplitudes of Rev-erba in C57 WD+LD/DD mice were notably repressed compared with those in C57 ND mice. The peak periodic value in C57 WD+LD/DD mice was significantly altered compared with that in control group. The 
mRNA levels were normalized to GAPDH. All the data were presented as mean \pm SD at ZT0, ZT4, ZT8, $\mathrm{ZT} 12, \mathrm{ZT} 16$, and ZT20 time-point ( $\mathrm{n}=4$ for each group), $*, \mathrm{P}<0.05, * *, \mathrm{P}<0.01$ versus $\mathrm{ZTO}$. (B) ChIP assay was performed to indicate whether Rev-erba could transcriptionally regulate the autophagy-related genes. There was no significant enrichment in Rev-erba to promote RORa as negative control, and Bmal1 was promoted as high as 9.3-fold as positive control. Besides, the autophagy-related genes, ULK1 and Atg5, demonstrated $\sim 3.45$ - and $~ 4.78$-fold enrichment of Rev-erba recruitment to their promoter, respectively. The mRNA levels were normalized to GAPDH. All the data were presented as mean \pm SD at ZT0, ZT4, ZT8, $\mathrm{ZT} 12, \mathrm{ZT} 16$, and ZT20 time-point. $\mathrm{n}=4$ for each group, $*, \mathrm{P}<0.05, * *, \mathrm{P}<0.01$ versus control. (C) Primary liver cells were isolated form mice, and we utilized siRNA to decrease the expression of Rev-erba. When the expression of Rev-erba decreased to 1.56-fold, the expressions of ULK1 and Atg5 were reduced to $\sim 0.96$ - and $\sim 1.19$-fold, respectively. The mRNA levels were normalized to GAPDH. All the data were presented as mean $\pm \mathrm{SD}$ ( $\mathrm{n}=4$ for each group), $*, \mathrm{P}<0.05, * *, \mathrm{P}<0.01$ versus control siRNA. 